\title{
Economic analysis of insulin initiation by pharmacists in a Canadian setting: The RxING study
}

Stephen Brown, PhD; Yazid N. Al Hamarneh, BSc(Pharm), PhD;

Ross T. Tsuyuki, BSc(Pharm), PharmD, MSc, FCSHP, FACC;

Kimberley Nehme, MSc; Luc Sauriol, MSc

\section{ABSTRACT}

Background: Conducted in Alberta, the RxING study examined the effect of a community pharmacist prescribing intervention on glycemic control in patients with uncontrolled type 2 diabetes mellitus (T2DM) using insulin glargine. The objective of this study was to assess the cost-effectiveness of pharmacists' prescribing of insulin glargine as an early intervention in uncontrolled patients with T2DM vs usual clinical practice.

Methods: The IMS CORE diabetes Markov model was used to project long-term clinical outcomes, costs and cost-effectiveness of interventions. The efficacy of insulin glargine, in terms of hemoglobin A1c reduction and hypoglycemia rates, was obtained from the RxING study. Health utility and cost data were found in Canadian publications.
The base-case analyses examined the economic and clinical effects of having pharmacists initiate insulin therapy in patients with uncontrolled T2DM in comparison to a physician initiate it up to 3 years later.

Results: Insulin initiation by pharmacists with uncontrolled T2DM patients is cost-effective. Having pharmacists prescribe insulin 1 year earlier than usual clinical practice resulted in an incremental cost savings of $\$ 805$ (CDN\$) and a gain of 0.048 QALYs per patient. Pharmacists prescribing insulin 2 years earlier resulted in an incremental cost savings of $\$ 624$ (CDN\$) per year and a gain of 0.075 quality-adjusted life-years (QALYs). Prescribing 3 years earlier allowed for a minor increase of $\$ 26$ and a gain of 0.086 QALYs.

Conclusion: Earlier initiation of insulin by pharmacists in uncontrolled T2DM patients, resulted in cost savings and delays in the development of diabetes-related complications, leading to an improved quality of life and increased survival rates. Can Pharm J (Ott) 2016;149:130-137.

\section{Introduction}

Type 2 diabetes mellitus (T2DM) is a progressive disease with at least $50 \%$ loss in insulin-producing capacity at the time of diagnosis, with an average loss of $5 \%$ annually thereafter. ${ }^{1}$ As such, many patients with T2DM will require insulin in order to maintain acceptable glycated hemoglobin (HbA1c) levels. Despite evidence from the Canadian INSIGHT study and the recommendations of the Canadian Diabetes Association guidelines, ${ }^{2}$ which demonstrate that initiating insulin glargine results in improved glycemic control, some physicians seem hesitant to prescribe it.

Pharmacists are frontline health care professionals who see patients with T2DM frequently and so could help patients achieve their targets by intervening in a timely manner. In Alberta, pharmacists can prescribe insulin and order 
laboratory tests. This advanced practice provides an opportunity for early identification and intervention in patients with uncontrolled T2DM. Recently, a Canadian study (the RxING study) aimed to determine the effect of a community pharmacist prescribing intervention on glycemic control in patients with poorly controlled T2DM. ${ }^{3}$ The RxING study showed pharmacists can help patients to significantly reduce patients' HbAlc by initiating basal insulin with insulin glargine. The RxING study reported that a community pharmacist prescribing intervention improved glycemic control in patients with uncontrolled T2DM. HbA1c was reduced from 9.1\% (SD 1) at baseline to $7.3 \%$ (SD 0.9) at 26 weeks, whereas fasting plasma glucose (FPG) was reduced from $11 \mathrm{mmol} / \mathrm{L}$ (SD 3.3) at baseline to $6.6 \mathrm{mmol} / \mathrm{L}$ (SD 1.8) at 26 weeks.

The objective of this study was to evaluate the cost-effectiveness of pharmacists' prescribing of insulin glargine as an early intervention in patients with uncontrolled T2DM in comparison to waiting for later insulin initiation, as is often seen in usual diabetes care. This economic study was designed in order to understand and examine the impact and benefits of early initiation of insulin glargine (Lantus, Sanofi, Quebec, Canada) therapy via an economic evaluation based on data from the RxING study.

\section{Methods}

This cost-effectiveness analysis compared 2 groups of patients with uncontrolled T2DM with similar characteristics: one group was the RxING study cohort, while the other one was a hypothetical cohort followed by a physician. We assumed the 2 groups had the same baseline characteristics. The group followed by pharmacists was switched immediately to insulin and was assumed to continue on this treatment regimen. In the hypothetical delayed cohort, described below, patients were switched to insulin 1 to 5 years later, upon consulting the physician, reflecting the current clinical inertia reported in recent studies. ${ }^{4,5}$

\section{Model overview}

Cost-utility analysis combines the incremental cost of the addition of insulin glargine to oral antidiabetic drugs (OADs) with the benefit it can bring in terms of quality and quantity of life, expressed as quality-adjusted life-years (QALYs). In addition, the model considers different costs and disutilities for future diabetes-related complications. The IMS CORE Diabetes Model was used to evaluate the costeffectiveness. Details of this model are described elsewhere. ${ }^{6}$ Simulations measured the costs and utilities associated with pharmacists' initiating insulin 2 years earlier than did the physician. It was assumed that patients would receive care from the same health care professional for the duration of the analysis.

A 50-year time horizon was considered to capture future diabetes complications during the patient's lifetime. We used a $5 \%$ discount rate. Only direct health care costs were considered in the model.

Direct medical costs, including treatment costs, pharmacy costs and medical costs related to the complications of having T2DM, were considered, as the perspective of this analysis was based on the Canadian health care system.

\section{Patients}

The IMS CORE Diabetes Model was populated based on the patients' characteristics from the RxING study. ${ }^{3}$ Default values from the IMS CORE Diabetes Model were retained if no data were available from the RxING study.

Characteristics that were not collected during the trial were taken from a previous study ${ }^{7}$ that economically evaluated third-line therapies in patients uncontrolled on the metforminsulfonylurea combination. In this study, patients' mean age was 64 years, and they had diabetes for 9.2 years, HbAlc of $9.1 \%$, body mass index of $29.4 \mathrm{~kg} / \mathrm{m}^{2}$ and a clinical diagnosis of T2DM for $\geq 6$ months (Table 1). ${ }^{3}$ The model also took into account the rates of major and minor hypoglycemic episodes, which were $0.623 \%$ and $17.245 \%$, respectively.

\section{Clinical efficacy}

As reported in the RxING study, pharmacists' initiation of insulin in patients with poorly controlled T2DM resulted in a 1.8\% (95\% confidence interval $[\mathrm{CI}], 1.4-2 ; p<0.001)$ absolute reduction in $\mathrm{HbAlc}$ levels, as well as a $4.1 \mathrm{mmol} / \mathrm{L}(95 \% \mathrm{CI}$, 3.3-5; $p=0.007)$ absolute reduction in $\mathrm{FPG}^{3}{ }^{3}$

\section{Resource use and cost data}

Drug-related costs. The costs of OADs, insulin, needles, self-monitored blood glucose test strips and lancets were based on Canadian prices and are described in Table 2. A markup of $10 \%$ was 
TABLE 1 Patient characteristics

\begin{tabular}{|c|c|c|c|}
\hline \multicolumn{4}{|l|}{ Patient demographics } \\
\hline Parameters & Mean & Unit & Reference \\
\hline Start age & 64 & Years & RxING study ${ }^{3}$ \\
\hline Duration of diabetes & 10.2 & Years & RxING study ${ }^{3}$ \\
\hline Male & 58 & $\%$ & \\
\hline \multicolumn{4}{|l|}{ Baseline risk factors } \\
\hline $\mathrm{HbA} 1 \mathrm{c}$ & 9.1 & $\%$ & RxING study ${ }^{3}$ \\
\hline SBP & 149 & $\mathrm{mmHg}$ & IMS CORE $^{6}$ \\
\hline $\mathrm{T}-\mathrm{CHOL}$ & 234 & $\mathrm{mg} / \mathrm{dL}$ & IMS CORE $^{6}$ \\
\hline HDL & 46 & $\mathrm{mg} / \mathrm{dL}$ & IMS CORE $^{6}$ \\
\hline LDL & 157 & $\mathrm{mg} / \mathrm{dL}$ & IMS CORE $^{6}$ \\
\hline TRIG & 162 & $\mathrm{mg} / \mathrm{dL}$ & IMS CORE $^{6}$ \\
\hline BMI & 31.6 & $\mathrm{~kg} / \mathrm{m}^{2}$ & RxING study ${ }^{3}$ \\
\hline$\%$ smoker & 22 & $\%$ & RxING study ${ }^{3}$ \\
\hline Cigarettes/day & 2 & - & IMS CORE $^{6}$ \\
\hline Alcohol consumption & 2 & oz/wk & RxING study ${ }^{3}$ \\
\hline \multicolumn{4}{|c|}{ Racial characteristics (\%) } \\
\hline White & 89 & $\%$ & RxING study ${ }^{3}$ \\
\hline Black & 5 & $\%$ & RxING study ${ }^{3}$ \\
\hline Hispanic & 0 & $\%$ & RxING study ${ }^{3}$ \\
\hline Native American & 1 & $\%$ & RxING study ${ }^{3}$ \\
\hline Asian/Pacific Islander & 5 & $\%$ & RxING study ${ }^{3}$ \\
\hline
\end{tabular}

BMI, body mass index; $\mathrm{HbA1c}$, hemoglobin A1c; HDL, high-density lipoprotein; LDL, low-density lipoprotein; SBP, systolic blood pressure, T-CHOL, total cholesterol; TRIG, triglyceride.

applied to the lowest available price, which also includes a $\$ 7.00$ pharmacy fee per 90 -day supply. ${ }^{7}$ Eligible patients remained on OADs, which included metformin (2000 $\mathrm{mg}$ ) and glyburide $(10 \mathrm{mg})$, until a switch occurred to insulin glargine. It was assumed that patients were receiving the maximum daily dose of metformin when switched to insulin glargine. ${ }^{8}$ Doses of glyburide were based on the defined daily dose (DDD) published by the World Health Organization Collaborating Centre for Drug Statistics Methodology. ${ }^{9}$ The daily dose of insulin glargine (31.1 units) used in the model was based on the RxING study. It was assumed that there would be no changes to the doses of insulin glargine (once titrated) and oral hypoglycemic agents. The use of lancets and test strips was also considered in the model. Patients using hypoglycemic agents, like insulin, typically test their blood glucose frequently; therefore, they use more test strips when compared to those on oral hypoglycemic agents. We assumed that patients on insulin would require 2.08 tests per day, whereas patients on oral hypoglycemic agents would require 1.16 tests per day. ${ }^{10}$

Costs due to diabetes complications. The costs of diabetes-related complications were obtained from various sources (Table 3 ) and expressed in 2014 Canadian dollars. All costs were inflated to 2014 using the health care component of the Canadian Consumer Price Index. ${ }^{8}$ 
TABLE 2 Insulin and glycemic test costs

\begin{tabular}{l|c|l}
\hline Item & Cost (\$CAN) & \multicolumn{1}{c}{ Reference } \\
\hline Insulin glargine (vial) & $\$ 60.52$ & Alberta cost ${ }^{11}$ \\
\hline Insulin glargine (cartridge, 1500 IU) & $\$ 91.39$ & Alberta cost $^{11}$ \\
\hline Syringes (per unit) & $\$ 0.22$ & ${\text { Harris et al. }(2007)^{12}}^{12}$ \\
\hline Glyburide (10 mg/day) & $\$ 0.11$ & Alberta cost $^{11}$ \\
\hline Metformin (2000 mg/day) & $\$ 0.23$ & Alberta cost $^{11}$ \\
\hline Dispensing fee & $\$ 7.00$ & Canadian Agency for Drugs and Technologies in Health report $^{7}$ \\
\hline Lancets (BD Ultra-Fine II Lancets) & $\$ 0.06$ & Diabetes Depot (www.diabetesdepot.ca) \\
\hline Test strips & $\$ 0.80$ & Canadian Agency for Drugs and Technologies in Health report $^{7}$ \\
\hline
\end{tabular}

Costs due to hypoglycemic episodes. It was assumed that mild/moderate hypoglycemic events required no health care resource use and as such had no associated costs. ${ }^{8}$ The cost of a severe hypoglycemia event was based on the 2013 CADTH report on optimal use of thirdline therapies. The cost of a severe event was estimated at \$2030.91 (\$CAN 2012). ${ }^{8}$

\section{Utility data}

Patients with T2DM who had no complications were assumed to have a utility value of $0.80 .{ }^{11}$ Disutilities for diabetes-related complications were obtained from the literature (Table 4).

\section{Sensitivity analysis}

In the sensitivity analysis, insulin initiation time by the physician varied (up to 5 years after the pharmacist), with the assumption that patients are initially followed by their pharmacist for the 1st year and by their physician thereafter.

\section{Results}

Pharmacists initiating insulin 2 years earlier than usual clinical practice resulted in a costsaving intervention in patients with uncontrolled T2DM on OADs. The resulting improvement in glycemic control triggered savings in preventing long-term complications, despite the increase in treatment cost. It resulted in cost savings of $\$ 624$ (CDN\$) and a gain of 0.075 QALYs and 0.090 lifeyears, making the early pharmacist intervention dominant over usual clinical practice. Initiation at 1 year earlier also resulted in greater savings (\$805) and an improvement of 0.048 QALYs.

When looking at longer time intervals (3-5 years earlier), we noticed that the results became cost-effective rather than cost-saving. At a 3-year interval, the cost difference was $\$ 26$ more for the pharmacist intervention, but the QALY gained remained higher than usual clinical practice (0.086 QALY gained), reaching an incremental cost-effectiveness ratio (ICER) of \$244/QALY. The trend was the same up to 5 years, with an increase in cost difference up to $\$ 766$, but also an increase in incremental QALY up to 0.101 . At year 5, the ICER increased to \$7613/QALY (Table 5). Although the ICER increased, it remained well under the $\$ 50,000$ threshold that is often used in Canada. $^{19}$

\section{Discussion}

The RxING study demonstrated that a pharmacist's prescribing of basal insulin in uncontrolled T2DM patients resulted in a $1.8 \%$ decrease in HbAlc. The findings of the RxING study are consistent with the findings of the Canadian INSIGHT study, which reported an absolute reduction of $1.55 \%$ in $\mathrm{HbA1c}$ when insulin was initiated by family physicians. ${ }^{20}$ Considering this finding, one would wonder about the impact on the ability of pharmacists to provide savings to the health care system. This economic analysis shows that for the first 2 years, having pharmacists initiate insulin earlier is a cost-saving intervention where benefits (mostly preventing diabetes-related long-term complications) offset the additional cost of insulin glargine.

The expectations were that the longer the delay in initiating basal insulin, the greater the clinical benefits and savings would be. This rang true regarding the clinical benefits, as more complications were avoided while improving the QALYs and life-years gained; however, it was 
TABLE 3 Complication costs related to type 2 diabetes mellitus

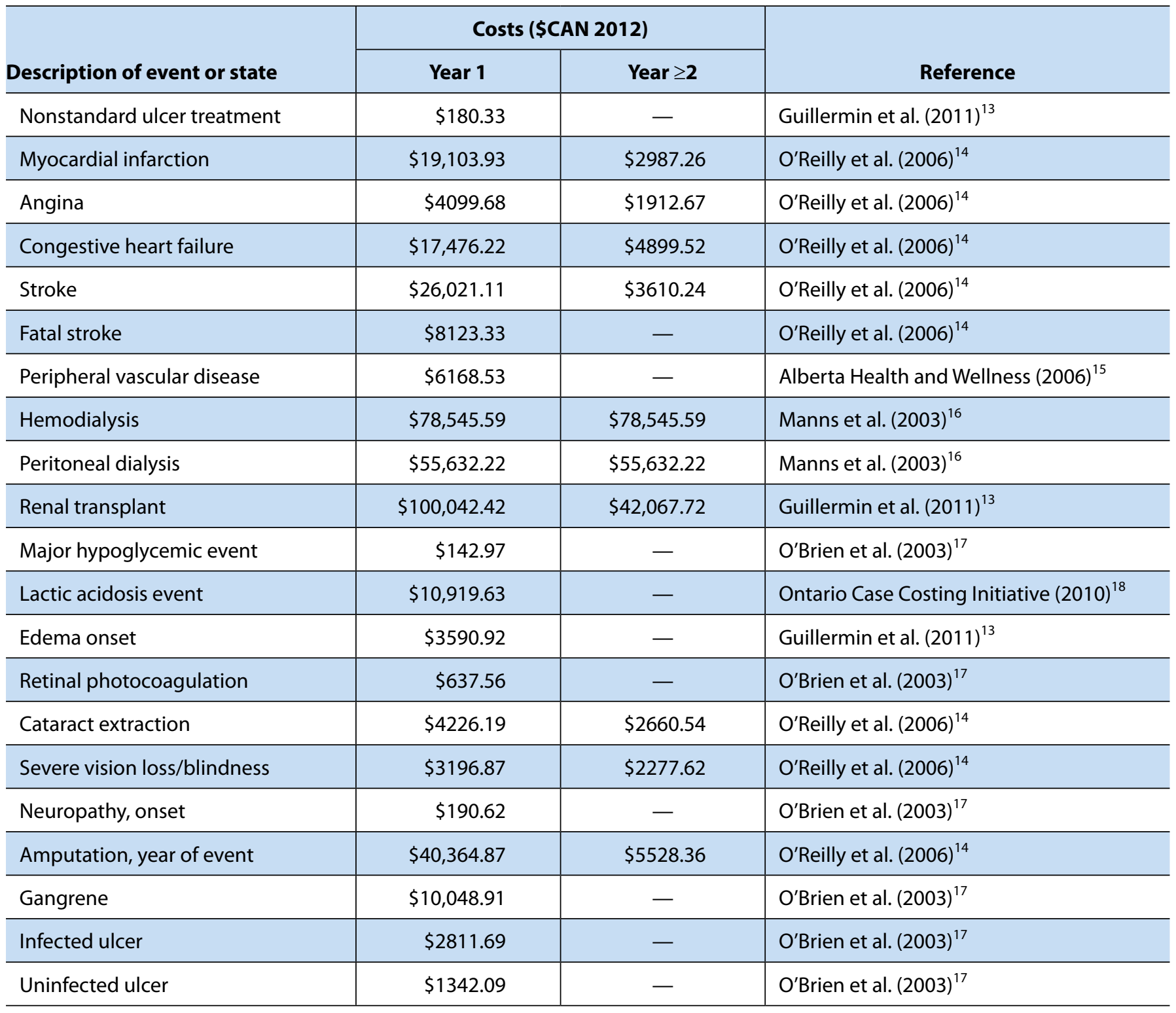

a different case when looking at costs. Results show that the intervention is cost-saving on a horizon of up to 2 years, while it becomes costeffective thereafter. The fact that it becomes costeffective instead of remaining cost-saving with an increasing delay is due to the fact that delaying insulin initiation reduces treatment costs but also maintains a suboptimal glycemic control in patients.

Increasing the delay in insulin therapy initiation is detrimental to glycemic control, increases the risk of long-term complications (while decreasing patients' quality of life) and goes against the main objective of the medical community, improving people's health. Moreover, relying on such a strategy to limit health care spending goes against the basis of patients' well-being and optimal diabetes treatment. Despite this, however, our results still show that insulin initiation by pharmacists is a desirable intervention, as it is either cost-saving or well within the proposed cost-effective threshold of $\$ 50,000 /$ QALY. The annual cost difference, even at a 5-year delay, remains marginal in light of the clinical benefits this can bring to patients in avoiding late insulin initiation.

This model, although robust, took into account some simplified assumptions regarding the treatment and care of patients with uncontrolled T2DM. For example, at insulin initiation, OAD treatment was not the same across all patients, as it was not an inclusion criterion; 
TABLE 4 Utilities and disutilities associated with type 2 diabetes complications

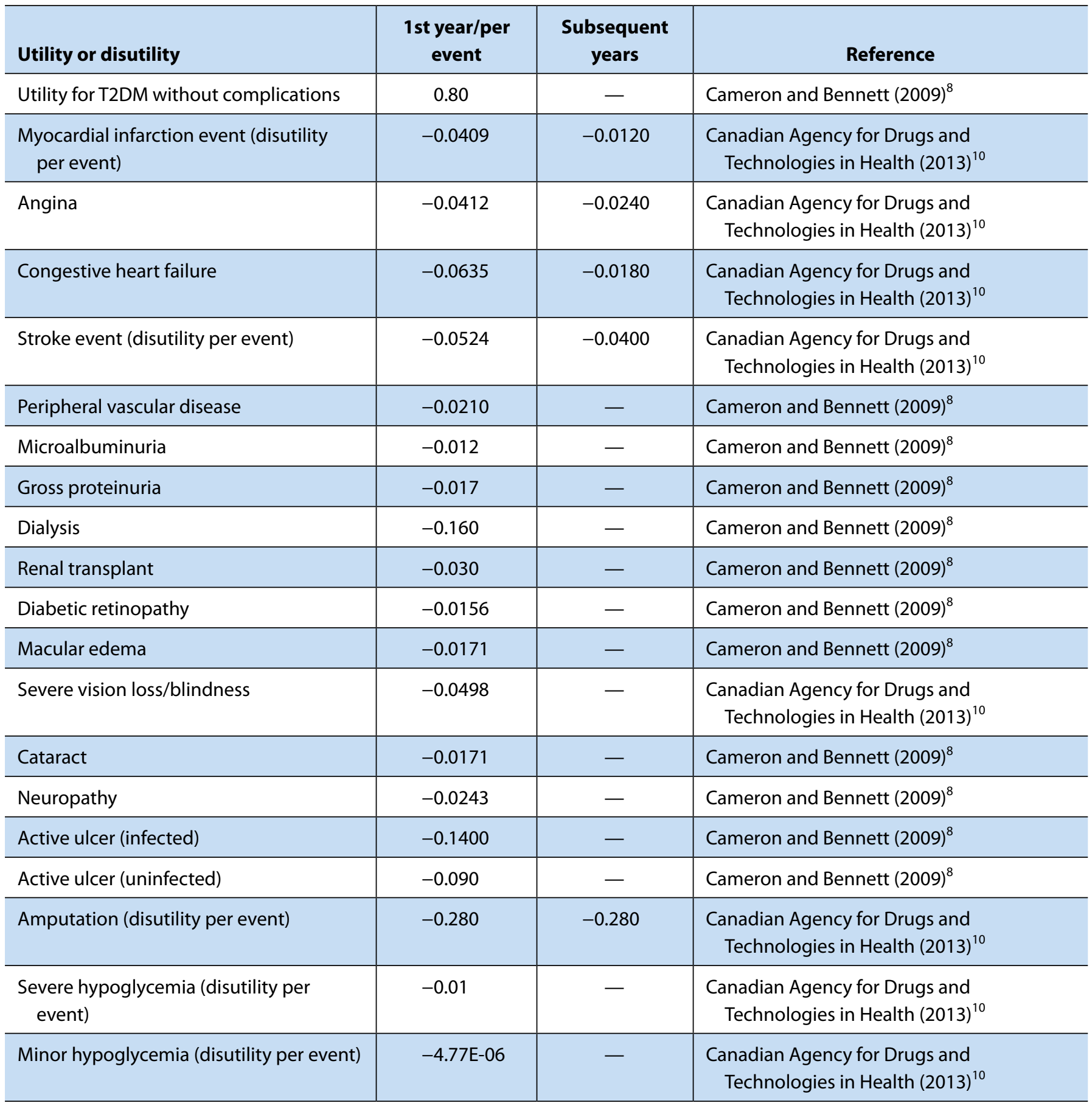

however, we do not expect this to have an impact on the economic results, as those agents were assumed to be the same for both comparative groups. We also assumed that patients were followed by either a pharmacist or a physician for diabetes management, while in reality, patients are usually managed by a health care team. The impact of having a 1st-year follow-up by pharmacists, and then carrying over to a physician, was assessed. Results were marginally affected due to the difference in professional fees for management plans and follow-up visits.

In the analysis, we did not account for potential OAD intensification in the delayed insulin initiation group. That would have a direct impact on costs (increasing costs in the usual care group) and glycemic control (increasing benefits or reduced complications in the usual care group). Similarly, in the model, we assumed no insulin dose adjustments once the 6-month 
TABLE 5 Cost-effectiveness of pharmacists' prescribing of insulin glargine as an early intervention in uncontrolled patients with type 2 diabetes mellitus vs usual clinical practice

\begin{tabular}{l|c|c|c|c|c}
\hline $\begin{array}{l}\text { Scenario (patient switching } \\
\text { to insulin after } \boldsymbol{x} \text { years) }\end{array}$ & $\boldsymbol{\Delta}$ Cost & $\boldsymbol{\Delta} \mathbf{Q A L Y s}$ & $\boldsymbol{\Delta} \mathbf{L Y s}$ & $\begin{array}{c}\text { Incremental } \\
\text { cost/QALY }\end{array}$ & $\begin{array}{c}\text { Incremental } \\
\text { cost/LY }\end{array}$ \\
\hline 1 year & $-\$ 805.00$ & 0.048 & 0.061 & Dominant & Dominant \\
\hline 2 years & $-\$ 624.00$ & 0.075 & 0.090 & Dominant & Dominant \\
\hline 3 years & $\$ 26.00$ & 0.086 & 0.104 & $\$ 297$ & $\$ 244$ \\
\hline 4 years & $\$ 677.00$ & 0.094 & 0.111 & $\$ 7240$ & $\$ 6100$ \\
\hline 5 years & $\$ 766.00$ & 0.101 & 0.116 & $\$ 7613$ & $\$ 6614$ \\
\hline
\end{tabular}

LY, life-year; QALY, quality-adjusted life-year.

titration was completed. This may have increased the cost of therapy but also may have improved the efficacy in the pharmacist group.

\section{Conclusion}

The findings of this analysis demonstrated the vital role that pharmacists can play in improving glycemic control in patients with uncontrolled
T2DM. Pharmacists' intervention for glycemic control in patients with uncontrolled T2DM being clinically beneficial in improving glycemic control projects reduced long-term complications while providing savings to the health care system. This could be an attractive approach considering current treatment gaps and the shortage of family physicians in certain areas in Canada.

From the Cornerstone Research Group Inc. (Brown), Burlington, Ontario; EPICORE Centre (Al Hamarneh, Tsuyuki), University of Alberta, Edmonton, Alberta; and Sanofi-Aventis Canada Inc. (Nehme, Sauriol), Laval, Quebec. Contact luc.sauriol@sanofi.com.

Author Contributions: Y. N. Al Hamarneh and R. T. Tsuyuki contributed to the RxING original study and to the writing of this manuscript. L. Sauriol, S. Brown and K. Nehme conducted the economic analyses and contributed to the writing of this manuscript. All authors approved the final version of the manuscript.

Declaration of Conflicting Interests: $L$. Sauriol and K. Nehme are employees of Sanofi-Aventis Canada Inc. S. Brown works for Cornerstone Research, and is a consultant who received a fee for running the analyses; as an employee of Cornerstone Research, S. Brown is under confidentiality with Sanofi regarding these analyses.

Funding: The study was funded by Sanofi-Aventis Canada Inc.

Data sharing:There are no additional data to be shared with this economic analysis other than the economic results presented in the article.

\section{References}

1. Lebovitz HE. Insulin secretagogues: old and new. Diabetes Rev 1999;7:139-53.

2. Canadian Diabetes Association Clinical Practice Guidelines Expert Committee. Pharmacologic management of type 2 diabetes. Can J Diabetes 2013;37:S61-8.

3. Al Hamarneh YN, Charrois T, Lewanczuk R, et al. Pharmacist intervention for glycaemic control in the community (the RxING study). BMJ Open 2013;3:e003154.

4. Harris SB, Kapor J, Lank CN, et al. Clinical inertia in patients with T2DM requiring insulin in family practice. Can Fam Physician 2010;56:e418-24.
5. Shah BR, Hux JE, Laupacis A, et al. Clinical inertia in response to inadequate glycemic control: do specialists differ from primary care physicians? Diabetes Care 2005;28:600-6. 6. Palmer AJ, Roze S, Valentine WJ, et al. The CORE Diabetes Model: projecting long-term clinical outcomes, costs and cost-effectiveness of interventions in diabetes mellitus (types 1 and 2) to support clinical and reimbursement decision-making. Curr Med Res Opin 2004;20(suppl 1):S5-26.

7. Canadian Agency for Drugs and Technologies in Health. CADTH therapeutic review: economic evaluation: thirdline therapy for patients with type 2 diabetes inadequately 
controlled with metformin and sulfonylurea combination therapy. August 2010. Available: https://www.cadth.ca/ sites/default/files/pdf/Diabetes_TR_Economic_Evaluation_ Final_e.pdf (accessed Mar 1, 2016).

8. Cameron CG, Bennett HA. Cost-effectiveness of insulin analogues for diabetes mellitus. CMAJ 2009;180:400

9. World Health Organization Collaborating Centre for Drug Statistics Methodology. ATC/DDD index 2106. Available: www.whocc.no/atc_ddd_index/ (accessed Jul. 20, 2014).

10. Canadian Agency for Drugs and Technologies in Health. Third-line pharmacotherapy for type 2 diabetes: update. CADTH Optimal Use Report 2013. Available: https://www. cadth.ca/sites/default/files/pdf/OP0512_Diabetes\%20 Update_Third-line_e.pdf (accessed Mar. 1, 2016).

11. Alberta Health. Interactive drug benefit list. Available: https:// idbl.ab.bluecross.ca/idbl/load.do (accessed Aug. 15, 2014).

12. Harris SB, Leiter LA, Yale JF, et al. Out-of-pocket costs of managing hyperglycemia and hypoglycemia in patients with type 1 diabetes and insulin-treated type 2 diabetes. Can J Diabetes 2007;31(1):25-33.

13. Guillermin AL, Samyshkin Y, Wright D, et al. Modeling the lifetime costs of insulin glargine and insulin detemir in type 1 and type 2 diabetes patients in Canada: a metaanalysis and a cost-minimization analysis. J Med Econ 2011;14:207-16.

14. O’Reilly D, Hopkins R, Blackhouse G, et al. Development of an Ontario Diabetes Economic Model (ODEM) and application to a multidisciplinary primary care diabetes management program. November 2006. Available: www.path-hta.ca/ Libraries/Reports/Development_of_an_Ontario_Diabetes_ Economic_Model_ODEM_and_Application_to_a_ Multidisciplinary_Primary_Care_Diabetes_Management_ Program.sflb.ashx (accessed Jul. 23, 2012).

15. Alberta Health and Wellness. Health costing in Alberta: 2006 annual report. Available: www.assembly.ab.ca/lao/ library/egovdocs/2006/alhw/129693_06.pdf (accessed Jul. 23, 2012).

16. Manns B, Lee H, Taub K, et al. Cost analysis of ongoing care of patients with end-stage renal disease: what are the important determinants? Edmonton (AB): Alberta Centre for Health Services Utilization Research; 2003.

17. O'Brien JA, Patrick AR, Caro JJ. Cost of managing complications resulting from type 2 diabetes mellitus in Canada. BMC Health Serv Res 2003;3:7.

18. Ontario Case Costing Initiative. OCCP website. 2010. Available: www.occp.com (accessed Jul. 23, 2012).

19. Laupacis A. Inclusion of drugs in provincial drug benefit programs: who is making these decisions, and are they the right ones? CMAJ 2002;166:44-7.

20. Gerstein HC, Yale J-F, Harris SB, et al. A randomized trial of adding insulin glargine vs avoidance of insulin in people with type 2 diabetes on either no oral glucose-lowering agents or submaximal doses of metformin and/or sulphonylureas. The Canadian INSIGHT (Implementing New Strategies with Insulin Glargine for Hyperglycaemia Treatment) Study. Diabet Med 2006;23:736-42. 